\title{
A Typical Development of Reading Skills in the Italian Primary School: Educational and Technological Perspectives
}

\author{
Stefano Di Tore \\ Ricercatore presso il Dipartimento di Scienze Umane Filosofiche e della Formazione dell'Università degli Studi di Salerno \\ Email: sditore@unisa.it
}

Sara Maffei

Dott.ssa in Scienze della Valutazione Motorio Sportiva e Tecniche di Analisi e Progettazione dello Sport per Disabili Email: sara.maffei81@gmail.com

\begin{abstract}
Maurizio Sibilio
Professore Ordinario presso il Dipartimento di Scienze Umane Filosofiche e della Formazione dell'Università degli Studi di Salerno Email: msibilio@unisa.it
\end{abstract}

Doi:10.5901/mjss.2016.v7n4p

Abstract

Results of the study on reading skills carried out in the "Programme for International Student Assessment" (PISA 2012) show that in Europe, on average, about $20 \%$ of fifteen-year-old students have very poor reading skills (levels detected in the "PISA TEST"). Italy fits perfectly into this scenario with a national average of about $19.5 \%$ of subjects aged fifteen with poor reading skills. The national average rises considerably in some regions such as Sicily, Calabria and Campania. Particularly, in the latter case the percentage of subjects with atypical development of reading skills is $28 \%$. The emergency situation described by the aforementioned study, taking into consideration the cheating phenomenon and the scientific debate on the evaluation in the Italian school system (Ferrer, 2013), led the University of Salerno to carry out a study with the aim of testing the reading levels using alternative standardized tests (MT test) and provide further data. The article presents the research findings conducted in 2015 in the region of Campania on the literacy levels of primary school pupils aged between 7 and 8 . The choice of this age group was also induced by the need to verify if this trend is also present at such a young age. The data seem to outline a critical situation regarding the development of reading skills. Results indicate that $32 \%$ of the sample requires some form of "immediate intervention" with the aim of levelling their reading skills to the expected standard for this age range. The data seem to be well supported by the results of other tests conducted by other institutions in the same territory². Moreover, they can be considered predictive of the future trend PISA test results have highlighted.

Keywords: Didattica, Tecnologia, Lettura, Inclusione

\section{Introduzione}

II linguaggio scritto, nelle sue due modalità di lettura e scrittura, costituisce probabilmente una delle tecnologie che maggiormente hanno influenzato il modo di strutturare la realtà da parte dell'uomo. La sua enorme diffusione per la generazione, la trasmissione e la conservazione delle informazioni lo rende uno degli strumenti cui siamo quotidianamente più esposti, determinandone in questo modo una quasi totale trasparenza (Di Tore, 2015). In altri termini, siamo così abituati all'uso di tale tecnologie da essere portati ad utilizzarla quasi come se si trattasse di un'abilità naturale. Tuttavia le competenze di lettura e scrittura sono competenze che non fanno parte del naturale sviluppo dell'individuo e che pertanto necessitano di essere apprese (Stella, 2004), e, in questo senso, il linguaggio scritto costituisce un importante focus di interesse didattico e pedagogico. II lavoro presentato nasce dall'analisi dei risultati di diversi monitoraggi sullo sviluppo della competenza di lettura effettuati nell'anno scolastico 2015-2016, che hanno messo in evidenza uno sviluppo atipico di tale abilità scolastica sul territorio campano (Italia). L'articolo procede presentando un quadro teorico di riferimento per la definizione della competenza di lettura, in seguito presenta i risultati dei monitoraggi effettuati, per poi concentrarsi sulle possibili metodologie didattiche inclusive applicabili all'interno della scuola primaria per favorire lo sviluppo della competenza di lettura. 


\section{La competenza di lettura}

II linguaggio scritto, può essere considerato come una strategia di adattamento semplessa o vicariante (Berthoz, 2011; Berthoz, 2015, Rivoltella 2014), sviluppata dall'uomo per organizzare, trasmettere e memorizzare le informazioni, per costruire e strutturare il pensiero e la conoscenza ecc. II linguaggio, in questa prospettiva, rappresenta una complessità accessoria in grado di semplessificare numerose attività dell'essere umano (Sibilio, 2014). La competenza di lettura costituisce una delle due modalità principali di tale strategia (lettura-scrittura), essa viene generalmente definita come un processo di transcodifica, ovvero come un processo di conversione da codice visivo a codice fonologico (Vio, 2013). Numerosi studi si sono focalizzati sulle abilità e sulle strategie cognitive coinvolte nel processo di lettura e sul loro sviluppo (Di Tore, 2014a), in questa sede verranno brevemente presentati i due modelli ad oggi maggiormente diffusi relativamente al funzionamento ed allo sviluppo cognitivo di tale competenza.

\section{II modello a due vie}

II modello a due vie costituisce, in ambito neuropsicologico, uno dei modelli più diffusi e consolidati relativamente ai processi cognitivi e neurali coinvolti nella lettura (Coltheart, 2001; Ludovico, 2015; Mangione, 2013; Di Tore, 2013; Di Tore 2014 b, Di Tore, 2014c; ).Il modello ipotizza l'esistenza di due differenti vie neurali, ognuna delle quali basata sull'interazione di diversi moduli cognitivi, attraverso le quali il processo di lettura può avere luogo (figura 1). Le due vie identificate all'interno del modello sono:

- La via lessicale-semantica-diretta: la parola da leggere è riconosciuta globalmente, attraverso la sua rappresentazione grafica, grazie al lessico ortografico di input (una sorta di dizionario in cui le parole sono immagazzinate nella loro forma grafica), il lessico ortografico di input invia l'output (la parola identificata) al sistema semantico, ove gli viene attribuito un particolare significato. L'output del sistema semantico (la parola identificata cui è stato attribuito uno specifico valore semantico) è poi utilizzato come input per iniziare un nuovo processo di ricerca all'interno del lessico fonologico in uscita - una sorta di dizionario in cui le parole sono immagazzinate nella loro forma fonologica. L'output del lessico fonologico in uscita viene quindi inviato al sistema articolatorio, permettendo la pronuncia della parola.

- La via fonologica-sublessicale-indiretta: l'output fono-articolato viene costituito attraverso l'associazione di grafemi e fonemi. La parola viene letta convertendo le singole unità grafiche in fonemi, attraverso un processo di fusione che prescindere dagli aspetti semantici, si ottiene in questo modo la rappresentazione fonologica della parola. La via fonologica-sublessicale-indiretta consente di leggere parole nuove o inventate (non-parole) (Cottini, 2008).

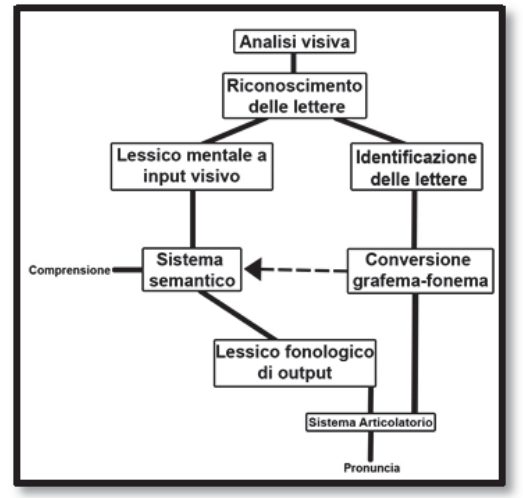

Figura 1: Schema "Modello a due vie" (Sartori, 1984)

Secondo il modello "il primo stadio d'analisi nella lettura è costituito dall'elaborazione sensoriale visiva della stringa di lettere e delle sue caratteristiche fisiche (luminanza, grandezza, colore, forma, orientamento), seguita dall'eventuale riconoscimento delle singole lettere". Da questo livello d'elaborazione partono due vie in parallelo: lessicale-diretta e fonologica-indiretta. Nella via lessicale il riconoscimento visivo dello stimolo avviene attraverso l'accesso al lessico ortografico d'ingresso. "Da lì è possibile accedere al sistema semantico che contiene i significati delle parole. II primo 
stadio della via fonologica permette l'accesso alla forma fonologica di uno stimolo (stringhe, non parole, oppure parole a bassa frequenza) secondo le regole di trasformazione grafema/fonema della lingua in uso. I principali disturbi di lettura osservati dai neuropsicologi sono stati interpreta ti come specifiche compromissioni delle varie vie della lettura postulate dal model lo standard" (Balconi, 2008, p. 92-93). Le due strategie di lettura descritte sono adoperate in modo spontaneo ed inconsapevole da tutti i normolettori. L'evidenza dell'esistenza di queste due vie di lettura è supportata dall'esistenza di pazienti con compromissioni specifiche di una sola di esse (Balconi, 2008, p. 92). Studi effettuati sembrano suggerire che, in soggetti normolettori, lo switch da una via all'altra in fase di lettura dipenda da alcuni parametri delle parole da leggere quali, frequenza d'uso, lunghezza, numero di vocali presenti etc (Tabella 1).

Tabella 1: la tabella riporta esempi di parole letti con strategie diverse. Mediamente il lettore tenderà a leggere le parole a sinistra attraverso la via lessicale-diretta e le parole a destra tramite la via sublessicale-indiretta (Vio, 2013).

\begin{tabular}{|c|c|}
\hline Lettura lessicale & Lettura fonologica \\
\hline Chiostro & Polimerizzazione \\
\hline Repubblica & Amitroptilina \\
\hline Gualdrappa & Novecentomilaquattrocentotredici \\
\hline
\end{tabular}

\section{II modello Uta-Frith}

il modello a due vie fornisce una prima descrizione del modo in cui le strategie di lettura operano, ma non fornisce informazioni circa i tempi e le tappe che scandiscono lo sviluppo della competenza di lettura. II modello Utah-Frith, presentato di seguito (figura 2), fornisce una schematizzazione dello sviluppo della competenza di lettura (Uta Frith, 1985).

II modello suddivide lo sviluppo della competenza di lettura in quattro fasi:

1. Fase logografica (4-5 anni): Nella fase logografica i soggetti percepiscono le parole nel loro insieme, come se fossero dei disegni. Sono in grado di distinguere ad esempio la parola "casa" dalla parola "cane" ma senza avere la consapevolezza che il grafema "c" della parola "casa" è lo stesso grafema "c" della parola "cane".

2. Fase alfabetica (4-5 anni): In questa fase i bambini sono in grado di leggere parole regolari (che non presentano differenze di conversione grafema fonema) e parole inventate (non-parole). In questa fase i soggetti utilizzano le competenze fonologiche per convertire grafemi in fonemi. La lettura è generalmente di tipo sillabico. I soggetti non sono ancora in grado di applicare al testo letto le regole ortografiche, essi non sono quindi in grado di leggere parole irregolari (come per esempio la parola "acqua" o "aglio").

3. Fase ortografica (7-8 anni): Durante questa fase il soggetto apprende la lettura delle parole irregolari, ovvero è in grado di leggere parole come "acqua" o "aglio" in modo corretto. Questa fase risulta particolarmente importante per le lingue opache (ovvero le lingue con una bassa corrispondenza fra "parlato" e "scritto", come ad esempio l'inglese 0 il francese) mentre risulta di minore importanza per le lingue trasparenti (come l'italiano o lo spagnolo).

4. Fase semantica (9-10 anni): In questa fase, il soggetto, è in grado di leggere, ad esempio, la parola "casa" senza doverla scomporre in fase di lettura nelle sillabe "ca" e "sa". Si sviluppa in questa fase la via lessicale diretta del modello a due vie. II "lessico visivo" ed il "lessico fonologico" dei soggetti cominciano in questa fase ad essere abbastanza sviluppati da poter consentire la lettura diretta di alcune parole.

\section{Figura 2:}

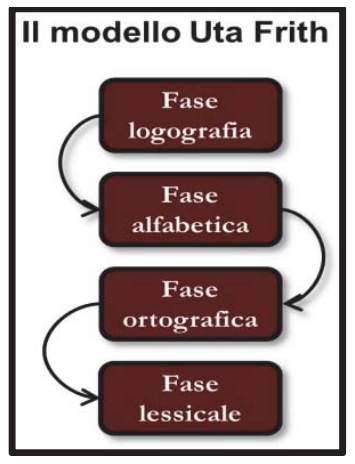




\section{La competenza di lettura}

II modello a "due vie" ed il modello Uta-Frith precedentemente esposti forniscono una descrizione delle strategie utilizzate in fase di lettura e del modo in cui la competenza di lettura si sviluppa. In relazione alla misurazione del grado di sviluppo della competenza di lettura è necessario ora introdurre come questa viene definita. La competenza di lettura si compone di due dimensioni principali:

1. Comprensione

2. Fluenza di lettura

La comprensione è relativa all'accesso al contenuto semantico del testo letto (quante informazioni essenziali il lettore riesce ad estrarre da testo dopo averlo letto). Viene misurata con una serie di domande relative al contenuto semantico del testo letto.

La fluenza di lettura è relativa alla competenza tecnica di lettura. Essa viene valutata sulla base di due parametri principali:

- Rapidità: è relativa alla velocità con cui un soggetto legge. Essa viene misurata con il numero di sillabe lette al secondo 0 con il numero di secondi impiegati a leggere una sillaba.

- Accuratezza: è relativa al numero ed alla tipologia di errori commessi durante la lettura. Viene misurata con un punteggio attribuito in base al numero ed alla tipologia di errori commessi.

I tre parametri utilizzati nella misurazione del grado di sviluppo della competenza di lettura possono potenzialmente avere andamenti indipendenti (un soggetto potrebbe avere uno scarso grado di comprensione del testo, ma avere un buon grado di sviluppo della rapidità e dell'accuratezza di lettura. Questo avviene, per esempio, in soggetti che presentano disturbi o patologie quali Iperlessia o Deficit da Comprensione del Testo), tuttavia la letteratura scientifica in materia suggerisce che mediamente i tre parametri menzionati sono fortemente correlati (generalmente soggetti con un elevata rapidità di lettura commettono meno errori, mentre soggetti con una rapidità di lettura bassa possiedono spesso anche un basso grado di comprensione e di accuratezza. La rapidità viene infatti considerata come un buon indice del grado di sviluppo dell'automatismo di lettura) (Tressoldi, 2001).

\section{Monitoraggi e raccolta dati}

II monitoraggio dello sviluppo della competenza di lettura all'interno della scuola primaria costituisce una delle finalità dalla legge n.170 dell'8 ottobre 2010 relativa ai Disturbi Specifici dell' Apprendimento (DSA). La legge sprona le istituzioni ad effettuare screening e monitoraggi al fine di poter identificare possibili situazioni a rischio di DSA. II monitoraggio della competenza di lettura risulta inoltre uno strumento efficace per valutare il livello di qualità dell'istruzione. Sulla base di queste premesse, sono stati effettuati nel corso dell'anno scolastico 2015-2016, 8 monitoraggi sullo sviluppo della competenza di lettura in 6 diverse scuole disseminate sul territorio campano. I monitoraggi sono stati effettuati fra il mese di novembre ed il mese di dicembre del 2015 ed hanno coinvolto un totale di 163 studenti frequentanti la terza classe della scuola primaria. Si precisa che i dati che saranno qui presentati sono relativi unicamente alle performances di studenti a sviluppo tipico (Non sono state elaborate le prove effettuate da soggetti con Bisogni Educativi Speciali, ovvero soggetti con minorazioni psico-fisiche, con Disturbi Specifici dell'Apprendimento, Italiano L-2, soggetti in situazione di svantaggio socio/economico/culturale. Le performances di soggetti con Bisogni Educativi Speciali non possono essere infatti valutate in relazione agli standard considerati).

\section{Strumenti}

I monitoraggi sono stati effettuati con le prove di lettura MT per le classi terza, quarta e quinta della scuola primaria. Le prove di lettura MT sono uno degli strumenti standardizzati più diffusi in Italia per la valutazione del livello di sviluppo della competenza di lettura. Esse sono costituite da un insieme di testi tarati, per lunghezza e difficoltà, sulle classi scolastiche di riferimento e sul periodo scolastico (per ogni classe di riferimento è prevista una prova di ingresso, una prova intermedia o una prova approfondita, una prova finale). Le prove sono suddivise in "prove di comprensione" e "prove di correttezza e rapidità". Le prime valutano attraverso una serie di domande relative al contenuto del testo il livello di comprensione del soggetto. Le seconde valutano la "fluenza di lettura" sulla base dei parametri di rapidità (numero di sillabe/secondi) e correttezza (numero e tipologia di errori commessi). Le prove forniscono inoltre degli standard di riferimento per la valutazione delle performances. Le prove utilizzate nei monitaggi (selezionate sulla base del periodo dell'anno e sulle classi cui sono state somministate) sono :

- Prova di comprensione: "L'asino nel fiume". 
- Prova di correttezza e rapidità: "l'idea più semplice".

Le prove MT forniscono inoltre un efficace sistema di scoring per l'attribuzione del punteggio. La tabella 3 riporta gli standard per la valutazione dei punteggi ottenuti nella prova "L'asino e il fiume" (tabella 3). I range di punteggio sono inoltre stati suddivisi in quattro fasce che permettono una valutazione immediata delle performances. Ogni fascia (contenente uno specifico range di punteggio) è riferita ad un criterio specifico per la valutazione della prova. I quattro criteri sono:

1. "criterio pienamente raggiunto";

2. "prestazione sufficiente rispetto al criterio";

3. "richiesta di attenzione";

4. "richiesta di attenzione immediata".

\section{Tabella 2:}

\begin{tabular}{|c|c|c|c|c|c|c|}
\hline \multicolumn{7}{|c|}{ Schema per la collocazione dei punteggi nelle prove di lettura } \\
\hline & Prova & Brano & $\begin{array}{l}\text { Criterio } \\
\text { pienamente } \\
\text { ragsiunto } \\
\end{array}$ & $\begin{array}{l}\text { Prestazione } \\
\text { sufficiente } \\
\text { rispetto al } \\
\text { criterio }\end{array}$ & $\begin{array}{l}\begin{array}{l}\text { Richiesta } \\
\text { attenzione }\end{array} \\
\end{array}$ & $\begin{array}{l}\text { Richiesta } \\
\text { intervento } \\
\text { immediato }\end{array}$ \\
\hline INTERMEDIA & $\begin{array}{l}\text { Comprensione } \\
\text { Correttezza } \\
\text { Rapidità }\end{array}$ & $\begin{array}{l}\text { Asino } \\
\text { L'idea } \\
\text { L'idea }\end{array}$ & $\begin{array}{l}9-10 \text { punti } \\
0-3 \text { errori } \\
>3.50\end{array}$ & $\begin{array}{l}7-8 \text { punti } \\
4-10 \text { errori } \\
3.50-1.83\end{array}$ & $\begin{array}{l}5-6 \text { punti } \\
11-15 \text { errori } \\
1.82-1.54\end{array}$ & $\begin{array}{l}0-4 \text { punti } \\
16 \text { errori e oltre } \\
<1.54\end{array}$ \\
\hline
\end{tabular}

Tabella 3:

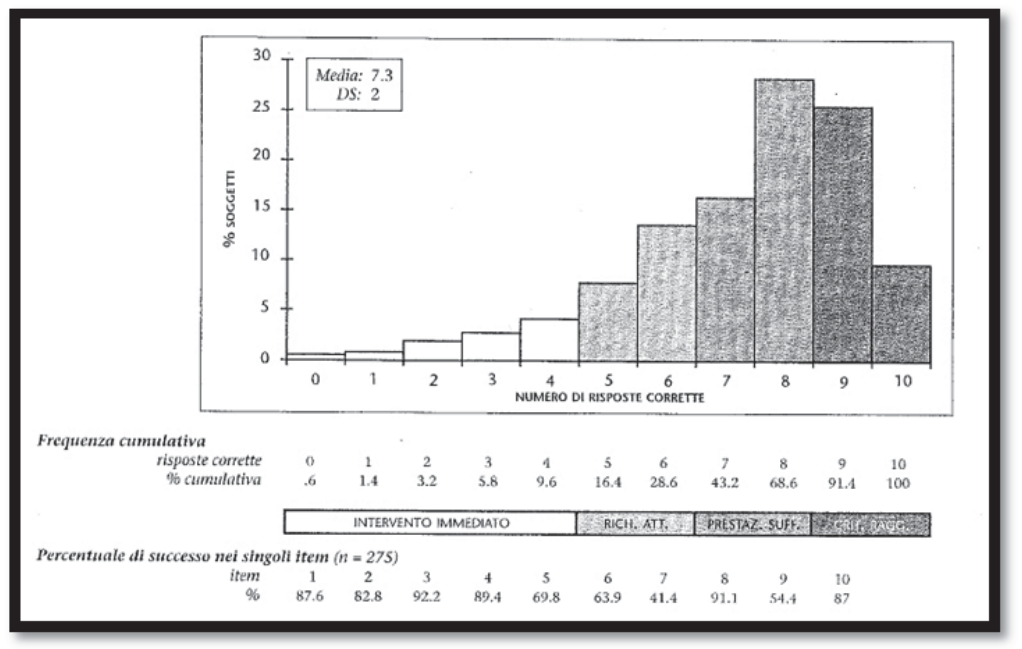

Si precisa che le indagini effettuate all'interno del programma PISA hanno adottato come strumento di valutazione le prove "PISA TEST", basate su task e statistiche di riferimento differenti rispetto alle "prove di lettura MT".

\section{Analisi dei dati}

La tabella 4 presenta i punteggi ottenuti dagli studenti alle prove "L'asino nel fiume" e "l'idea più semplice".

La tabella 4 presenta le seguenti colonne:

- ID: numero identificativo attribuito allo studente;

- Età: età dello studente in anni;

- Sesso: sesso dello studente; 
- Comprensione: Numero di risposte corrette fornite alla prova di comprensione "L'asino e il fiume"( la prova contiene in tutto 10 domande);

- Rapidità: Numero di sillabe lette mediamente al secondo nella prova "L'idea più semplice" (totale delle sillabe 289);

- Accuratezza: Punteggio attribuito al soggetto sulla base del numero e della tipologia di errori commessi in fase di lettura della prova "L'idea più semplice" (vedi tabella xxx per approfondimenti sull'attribuzione del punteggio di accuratezza).

\section{Tabella 4:}

\begin{tabular}{|c|c|c|c|c|c|c|c|c|c|c|c|}
\hline ID & Età & Sesso & Comprensione & Rapidità & Accuratezza & ID & Età & Sesso & Comprensione & Rapidità & Accuratezza \\
\hline 1 & 7 & $\mathrm{M}$ & 7 & 2,06 & 4,5 & 83 & 8 & M & 4 & 3,06 & 8 \\
\hline 2 & 8 & $M$ & 8 & 3,75 & 2 & 84 & 8 & $M$ & 7 & 2,35 & 4,5 \\
\hline 3 & 8 & $\mathrm{~F}$ & 7 & 2,53 & 5 & 85 & 8 & $F$ & 6 & 3,01 & 7,5 \\
\hline 4 & 8 & $\mathrm{M}$ & 3 & 1,4 & 5,5 & 86 & 8 & $\mathrm{M}$ & 8 & 2,89 & 4 \\
\hline 5 & 7 & M & 5 & 1,29 & 10 & 87 & 8 & $\mathrm{~F}$ & 9 & 2,58 & 5,5 \\
\hline 6 & 8 & $\mathrm{~F}$ & 7 & 1,94 & 5 & 88 & 8 & $\mathrm{M}$ & 6 & 2,02 & 14 \\
\hline 7 & 8 & M & 5 & 1,31 & 8,5 & 89 & 8 & M & 8 & 3,4 & 5 \\
\hline 8 & 7 & M & 7 & 1,59 & 14 & 90 & 8 & $\mathrm{~F}$ & 4 & 1,96 & 4,5 \\
\hline 9 & 8 & $\mathrm{~F}$ & 8 & 2,24 & 3,5 & 91 & 8 & M & 3 & 1,38 & 11 \\
\hline 10 & 8 & M & 5 & 1,88 & 13,5 & 92 & 8 & $\mathrm{~F}$ & 5 & 1,77 & 19 \\
\hline 11 & 7 & $\mathrm{M}$ & 6 & 1,81 & 7 & 93 & 7 & $\mathrm{~F}$ & 8 & 2,83 & 9 \\
\hline 12 & 7 & $\mathrm{M}$ & 7 & 2,19 & 2 & 94 & 8 & $\mathrm{~F}$ & 6 & 2,03 & 10 \\
\hline 13 & 8 & $\mathrm{~F}$ & 8 & 1,99 & 6,5 & 95 & 7 & $\mathrm{~F}$ & 10 & 3,44 & 1 \\
\hline 14 & 8 & $\mathrm{~F}$ & 8 & 2,92 & 0 & 96 & 8 & $\mathrm{~F}$ & 7 & 1,96 & 0,5 \\
\hline 15 & 8 & $\mathrm{~F}$ & 8 & 0,6 & 14,5 & 97 & 7 & $F$ & 8 & 3,36 & 1,5 \\
\hline 16 & 8 & $\mathrm{~F}$ & 7 & 1,58 & 10,5 & 98 & 8 & $\mathrm{~F}$ & 4 & 1,79 & 9 \\
\hline 17 & 7 & $\mathrm{M}$ & 8 & 1,51 & 2 & 99 & 8 & $\mathrm{~F}$ & 5 & 2,58 & 6 \\
\hline 18 & 8 & $F$ & 9 & 1,81 & 4 & 100 & 7 & $F$ & 8 & 3,07 & 4,5 \\
\hline 19 & 7 & $\mathrm{M}$ & 7 & 0,83 & 7 & 101 & 8 & $F$ & 7 & 2,67 & 2,5 \\
\hline 20 & 7 & $M$ & 6 & 2,11 & 2 & 102 & 8 & $F$ & 7 & 2,29 & 10 \\
\hline 21 & 7 & $M$ & 5 & 1,56 & 11 & 103 & 8 & $M$ & 5 & 1,88 & 2 \\
\hline 22 & 7 & $F$ & 5 & 1,7 & 4 & 104 & 7 & $M$ & 5 & 1,31 & 14,5 \\
\hline 23 & 8 & $\mathrm{M}$ & 0 & 1,03 & 9 & 105 & 7 & $F$ & 5 & 2,09 & 5 \\
\hline 24 & 8 & $F$ & 5 & 2,45 & 6 & 106 & 7 & $F$ & 7 & 2,89 & 4,5 \\
\hline 25 & 8 & $\mathrm{M}$ & 6 & 1,98 & 3,5 & 107 & 8 & $F$ & 5 & 1,86 & 15,5 \\
\hline 26 & 8 & $F$ & 6 & 2,58 & 4 & 108 & 8 & $F$ & 9 & 2,95 & 6 \\
\hline 27 & 8 & $M$ & 0 & 0 & 20 & 109 & 8 & $F$ & 8 & 2,12 & 13 \\
\hline 28 & 8 & $M$ & 8 & 3,17 & 1,5 & 110 & 7 & $M$ & 5 & 1,51 & 8 \\
\hline 29 & 8 & $M$ & 6 & 2,53 & 5 & 111 & 7 & $M$ & 3 & 1,28 & 20 \\
\hline 30 & 8 & $M$ & 0 & 0 & 20 & 112 & 8 & $\mathrm{M}$ & 2 & 3,42 & 3 \\
\hline 31 & 8 & $\mathrm{M}$ & 6 & 3,48 & 6 & 113 & 8 & $F$ & 5 & 2,47 & 4 \\
\hline 32 & 7 & $M$ & 5 & 1,96 & 6 & 114 & 8 & $\mathrm{M}$ & 5 & 1,79 & 4,5 \\
\hline 33 & 8 & $M$ & 5 & 2,03 & 13 & 115 & 8 & $F$ & 5 & 1,31 & 14,5 \\
\hline 34 & 8 & $\mathrm{~F}$ & 6 & 2,58 & 2 & 116 & 7 & $F$ & 6 & 2,09 & 8 \\
\hline 35 & 7 & $\mathrm{M}$ & 3 & 1,38 & 8,5 & 117 & 7 & $M$ & 5 & 1,49 & 10,5 \\
\hline 36 & 8 & $M$ & 4 & 3,11 & 3 & 118 & 8 & $F$ & 6 & 2,14 & 8,5 \\
\hline 37 & 8 & $\mathrm{M}$ & 5 & 2,14 & 4 & 119 & 7 & $\mathrm{M}$ & 3 & 1,45 & 13 \\
\hline 38 & 7 & $M$ & 5 & 1,73 & 3,5 & 120 & 7 & $F$ & 7 & 3,65 & 2 \\
\hline 39 & 8 & $F$ & 6 & 2,53 & 0 & 121 & 8 & $F$ & 6 & 2,01 & 7 \\
\hline 40 & 8 & $F$ & 6 & 2,29 & 6 & 122 & 7 & $F$ & 6 & 2,68 & 7 \\
\hline 41 & 8 & $M$ & 8 & 2,31 & 11 & 123 & 7 & $F$ & 3 & 1,36 & 10 \\
\hline 42 & 7 & $\mathrm{~F}$ & 7 & 2,75 & 7 & 124 & 7 & $\mathrm{M}$ & 2 & 1,33 & 11 \\
\hline 43 & 8 & $M$ & 5 & 1,86 & 14 & 125 & 8 & $M$ & 9 & 3,42 & 5 \\
\hline 44 & 8 & $F$ & 3 & 1,45 & 6 & 126 & 8 & $\mathrm{~F}$ & 5 & 2,21 & 4 \\
\hline 45 & 8 & $M$ & 5 & 1,63 & 9 & 127 & 8 & $M$ & 4 & 1,49 & 14 \\
\hline
\end{tabular}




\begin{tabular}{|c|c|c|c|c|c|c|c|c|c|c|c|}
\hline ID & Età & Sesso & Comprensione & Rapidità & Accuratezza & ID & Età & Sesso & Comprensione & Rapidità & Accuratezza \\
\hline 46 & 8 & $F$ & 5 & 2,29 & 5,5 & 128 & 7 & $F$ & 8 & 2,71 & 16 \\
\hline \begin{tabular}{|l|}
47 \\
\end{tabular} & 8 & $M$ & 3 & 1,44 & 16,5 & 129 & 7 & $M$ & 5 & 1,97 & 7,5 \\
\hline 48 & 8 & $F$ & 2 & 1,58 & 4 & 130 & 7 & $M$ & 6 & 2,77 & 4 \\
\hline 49 & 8 & $M$ & 5 & 2,05 & 11 & 131 & 8 & $F$ & 2 & 1,25 & 20 \\
\hline 50 & 8 & $F$ & 9 & 2,33 & 5 & 132 & 8 & $F$ & 6 & 2,32 & 4 \\
\hline 51 & 8 & $F$ & 8 & 1,36 & 9,5 & 133 & 7 & $\mathrm{~F}$ & 4 & 1,67 & 14 \\
\hline 52 & 8 & $M$ & 5 & 1,64 & 5 & 134 & 8 & $M$ & 3 & 1,34 & 12,5 \\
\hline 53 & 8 & $M$ & 8 & 2,58 & 15 & 135 & 8 & $M$ & 5 & 1,82 & 10,5 \\
\hline 54 & 7 & $M$ & 8 & 1,95 & 9 & 136 & 8 & $F$ & 6 & 2,62 & 5 \\
\hline 55 & 8 & $F$ & 7 & 3,07 & 4 & 137 & 7 & $M$ & 2 & 1,29 & 3,5 \\
\hline 56 & 8 & $M$ & 9 & 2,95 & 10,5 & 138 & 7 & $F$ & 8 & 3,4 & 3,5 \\
\hline 57 & 8 & $M$ & 8 & 2,37 & 9 & 139 & 8 & $M$ & 7 & 2,45 & 6,5 \\
\hline 58 & 7 & $M$ & 7 & 2,11 & 8 & 140 & 8 & $M$ & 3 & 1,06 & 10 \\
\hline 59 & 8 & $F$ & 6 & 1,32 & 15,5 & 141 & 7 & $F$ & 5 & 2,89 & 4 \\
\hline 60 & 8 & $F$ & 6 & 2,06 & 5 & 142 & 7 & $F$ & 2 & 1,15 & 13 \\
\hline 61 & 7 & $M$ & 1 & 0,61 & 20 & 143 & 8 & $F$ & 3 & 1,97 & 15 \\
\hline 62 & 7 & $M$ & 3 & 1,42 & 8 & 144 & 8 & $M$ & 5 & 1,59 & 17 \\
\hline 63 & 8 & $\mathrm{M}$ & 5 & 2,17 & 6 & 145 & 7 & $M$ & 7 & 2,51 & 4 \\
\hline 64 & 7 & $M$ & 9 & 3,32 & 5 & 146 & 8 & $F$ & 5 & 1,5 & 12 \\
\hline 65 & 8 & $F$ & 9 & 2,98 & 2,5 & 147 & 7 & $F$ & 7 & 2,49 & 1 \\
\hline 66 & 8 & $M$ & 9 & 1,59 & 14 & 148 & 8 & $\mathrm{M}$ & 5 & 2,62 & 3 \\
\hline 67 & 8 & $F$ & 6 & 1,88 & 20 & 149 & 8 & $F$ & 9 & 3,34 & 5 \\
\hline 68 & 8 & $M$ & 2 & 1,44 & 13 & 150 & 8 & $M$ & 6 & 2,16 & 8,5 \\
\hline 69 & 7 & $F$ & 8 & 2,35 & 10,5 & 151 & 7 & $M$ & 5 & 1,69 & 20 \\
\hline 70 & 8 & $M$ & 3 & 1,31 & 20 & 152 & 8 & $\mathrm{M}$ & 5 & 1,9 & 8 \\
\hline 71 & 8 & $M$ & 6 & 2,58 & 4,5 & 153 & 8 & $F$ & 6 & 2,66 & 5 \\
\hline 72 & 8 & $M$ & 6 & 2,56 & 7,5 & 154 & 8 & $F$ & 7 & 2,79 & 5 \\
\hline 73 & 7 & $F$ & 8 & 2,78 & 5 & 155 & 8 & $M$ & 5 & 1,8 & 7,5 \\
\hline 74 & 8 & $F$ & 7 & 3,8 & 3,5 & 156 & 7 & $F$ & 6 & 2,71 & 5,5 \\
\hline 75 & 7 & $F$ & 3 & 1,27 & 18 & 157 & 8 & $F$ & 4 & 1,37 & 4,5 \\
\hline 76 & 8 & $M$ & 3 & 1,21 & 20 & 158 & 8 & $M$ & 5 & 1,84 & 10 \\
\hline 77 & 8 & $M$ & 6 & 2,49 & 5 & 159 & 8 & $F$ & 5 & 2,47 & 5 \\
\hline 78 & 7 & $M$ & 4 & 1,76 & 14,5 & 160 & 7 & $M$ & 6 & 2,21 & 6 \\
\hline 79 & 8 & $F$ & 3 & 1,25 & 13,5 & 161 & 7 & $M$ & 8 & 3,51 & 4 \\
\hline 80 & 8 & $F$ & 8 & 2,65 & 5,5 & 162 & 8 & $M$ & 5 & 1,91 & 19 \\
\hline 81 & 8 & $F$ & 9 & 2,63 & 2 & 163 & 8 & $\mathrm{M}$ & 5 & 1,98 & 15 \\
\hline 82 & 8 & M & 10 & 3,7 & 2 & & & & & & \\
\hline
\end{tabular}

Tabella 5:

\begin{tabular}{|c|c|c|c|}
\hline & Comprensione & Rapidità & Accuratezza \\
\hline Media & 5,71 & 2,13 & 8,14 \\
\hline DS & 2,07 & 0,72 & 5,16 \\
\hline Rango percentile & 22,5 & 35 & 26.9 \\
\hline
\end{tabular}

Come è possibile notare nella tabella 4, per quanto riguarda i parametri di:

- Comprensione, i soggetti hanno ottenuto una media di 5,52 risposte corrette. II punteggio medio si inserisce nella fascia di "richiesta di attenzione" e si attesta notevolmente al di sotto della media nazionale $\left(22.5^{\circ}\right.$ percentile).

- Rapidità, la rapidità media rilevata è di 2,13 sillabe lette al secondo. La rapidità rientra in questa caso all'interno della fascia "prestazione sufficiente rispetto al criterio". II valore si attesta al di sotto della media nazionale ( $35^{\circ}$ percentile) (Tressoldi, 2001).

- Accuratezza, il punteggio medio rilevato è di 8,13. II punteggio medio rientra all'interno della fascia "Prestazione sufficiente". Il valore si attesta al di sotto della media nazionale ( $26.9^{\circ}$ percentile).

La Tabella 5 presenta la statistica descrittiva dei dati presentati. 
La tabella 6 illustra la percentuale di prestazioni esaminate che si attesta nella fascia "Richiesta di intervento immediato"(la fascia contiene i punteggi fra il $1^{\circ}$ e il $5^{\circ}$ percentile, una prestazione rientrante in questa indica la potenziale presenza di patologie o disturbi).

\section{Tabella 6:}

\begin{tabular}{|c|c|c|c|}
\hline & Comprensione & Rapidità & Accuratezza \\
\hline Richiesta di intervento immediato & $21,3 \%$ & $22,5 \%$ & $10 \%$ \\
\hline
\end{tabular}

La tabella 7 presenta le correlazioni rinvenute fra i punteggi rilevati in relazione ai parametri di comprensione, rapidità ed accuratezza.

\section{Tabella 7:}

\begin{tabular}{|c|c|}
\hline & Correlazione $(\mathrm{R})$ \\
\hline Comprensione/Rapidità & 0,65 \\
\hline Comprensione/Accuratezza & $-0,45$ \\
\hline Rapidità/Accuratezza & $-0,61$ \\
\hline
\end{tabular}

Come è possibile notare dai valori riportati nella tabella 7 , i parametri presentano fra di loro un livello di correlazione medio. In particolare la rapidità presenta una correlazione diretta con la comprensione ed inversa con l'accuratezza. In altri termini all'incrementarsi della rapidità di lettura corrisponde mediamente un incremento della comprensione del testo ed un decremento degli errori commessi. I parametri descritti nella tabella 7 appaiono in linea con gli studi relativi alla correlazione fra i tre valori (Stella, 2003).

\section{Discussioni}

I dati presentati sembrano indicare uno sviluppo anomalo della competenza di lettura in relazione alle scuole analizzate. In accordo con i criteri di valutazione delle prove $\mathrm{MT}^{1}$ il $32 \%$ del campione necessita di un "intervento immediato", volto a ricondurne le prestazioni all'interno di range di normalità. I dati presentati appaiono inoltre in linea con i risultati del progetto "Sviluppo tipico ed atipico della lettura nella provincia di Salerno" 2 condotto nel 2014. Nonostante la differenza in termini di fascia di età analizzata (rispettivamente 15 e 8 anni) e di strumenti utilizzati (rispettivamente PISA Test e Prove di lettura MT) il monitoraggio ha in sintesi espresso dati sostanzialmente coerenti con i risultati dell'indagine PISA. Ulteriori indagini saranno condotte per individuare le cause dei 4 punti percentuali di variazione individuati dalle due indagini (questi potrebbero essere attribuiti alla differenza delle fasce di età analizzate, agli strumenti utilizzati dai due studi, al fenomeno del cheating in relazione all'indagine PISA o all'esiguità del campione analizzato nel presente studio). I risultati del monitoraggio descrivono comunque una situazione emergenziale che necessita di adeguati interventi sociali, culturali e didattico-educativi.

\section{Prospettive}

Come descritto, la situazione emersa dall'analisi dei dati appare critica. Per quanto riguarda gli sviluppi futuri, attualmente si sta provvedendo ad individuare i soggetti che hanno avuto prestazioni rientranti all'interno della fascia "Richiesta di intervento immediato", al fine di suggerire loro una visita medica per valutare l'eventuale presenza di disturbi o patologie. Una volta terminata questa fase e scremato il campione selezionato da eventuali soggetti con disturbi o patologie non ancora certificate 0 diagnosticate, gli studenti individuati saranno sottoposti a delle analisi più approfondite volte ad identificare la natura del problema. Studi futuri saranno quindi indirizzati:

- all'estensione del campione di ricerca, con l'obiettivo di comprendere se il trend descritto può essere considerato come rappresentativo dell'intero territorio salernitano. Si intende inoltre estendere il campione anche al di fuori della provincia salernitana e dell'ambito territoriale campano, con lo scopo di analizzare la

\footnotetext{
${ }^{1}$ Cornoldi, C., \& Colpo, G. (1998). Prove di lettura MT per la scuola elementare. 2: manuale. OS.

2 Retrieved from: http://trasparenza.provincia.salerno.it/archivio28_provvedimenti_1_38422_725_1.html 
reale ampiezza del fenomeno.

- a comprendere le cause che hanno generato lo stato descritto e ad agire di conseguenza;

- alla realizzazione di metodologie e strumenti didattici inclusivi (Di tore, 2014) atti a favorire lo sviluppo della competenza di lettura all'interno della scuola primaria.

Per quanto riguarda la terza tipologia di studi, si precisa, nella piena consapevolezza di non poter ritenere le informazioni di seguito descritte come scientificamente affidabili, che la maggioranza degli insegnanti coinvolti nei monitoraggi, interrogata relativamente alle possibili cause della situazione, ha lamentato la mancanza di metodologie e strumenti didattici adeguati.

\section{References}

Balconi, M. (2008). Neuropsicologia della comunicazione [Neuropsychology of communication], Milano: Springer.

Berthoz, A. (2011). La semplessità [Simplexity], Codice.

Berthoz, A. and S. Ferraresi (2015). La vicarianza. II nostro cervello creatore di mondi [Vicariance. Our brain as creator of worlds], Codice.

Coltheart, M., Rastle, K., Perry, C., Langdon, R., \& Ziegler, J. (2001). DRC: a dual route cascaded model of visual word recognition and reading aloud. Psychological review, 108(1), 204.

Cottini, L. (2008). Per una didattica speciale di qualità: dalla conoscenza del deficit all'intervento inclusivo [Quality special education: from knowledge about the impairment to the inclusive intervention], Morlacchi.

Di Tore, P. A. (2014c). Perception Of Space, Empathy And Cognitive Processes: Design Of A Video Game For The Measurement Of Perspective Taking Skills. iJET, 9(7), 23-29.

Di Tore, P. A., Di Tore, S., Mangione, G. R., \& Ludovico, L. A. (2014a, September). MADRIGALE: A Multimedia Application for Dyslexia and Reading Improvement GAmifying Learning Experience. In Intelligent Networking and Collaborative Systems (INCoS), 2014 International Conference on (pp. 486-491). IEEE.

Di Tore, P. A., Discepolo, T., \& Di Tore, S. (2013). Natural User Interfaces as a powerful tool for courseware design in Physical Education. Journal of e-Learning and Knowledge Society, 9(2).

Di Tore, S., Fulgione, M., \& Sibilio, M. (2014b). Dislessia e Videogames: II Potenziale Didattico dei Videogiochi. Mediterranean Journal of Social Sciences, 5(23), 1165.

Di Tore, S.; Fulgione, M., Sibilio, M.(2014). Dislessia e videogames: II potenziale didattico dei videogiochi [Dyslexia and videogames: The educational potential of videogames] DOI:10.5901/mjss.2014.v5n23p1165. pp.1165-1171. In MEDITERRANEAN JOURNAL OF SOCIAL SCIENCES - ISSN:2039-9340 vol. 5 (23)

Di Tore, S.; Sibilio, M. (2015) L'invisibile tecnologia della parola: design e sviluppo di un software per dislessici [Invisible technology of the word: design and development of a software for dyslexics]. pp.33-41. In TD TECNOLOGIE DIDATTICHE - ISSN:1970-061X vol. 23(1)

Ferrer-Esteban, G. (2013). II 'cheating' nel sistema italiano di valutazione [Cheating in the evaluation of the Italian system], In Workshop 'Metodi di identificazione, analisi e trattamento del cheating' [Identification methods, analysis and treatment of cheating] , Rome, February (Vol. 8).

Frith, U. (1985). Beneath the surface of developmental dyslexia. Surface dyslexia, 32.

Ludovico, L. A., Di Tore, P. A., Mangione, G. R., Di Tore, S., \& Corona, F. (2015). Measuring the Reading Abilities of Dyslexic Children through a Visual Game. International Journal of Emerging Technologies in Learning (iJET), 10(7), 47-54.

Mangione, G. R., Discepolo, T., Di Tore, P. A., Di Tore, S., Cozzarelli, C., \& Corona, F. (2013, July). Measuring empathy to support learning design and narrative game: A phenomenological approach. In Complex, Intelligent, and Software Intensive Systems (CISIS), 2013 Seventh International Conference on (pp. 401-406). IEEE.

Rivoltella, P. C. (2014). La previsione. Neuroscienze, apprendimento, didattica, La Scuola.

Sartori, G., Barry, C., \& Job, R. (1984). Phonological dyslexia: A review. In Dyslexia: A global issue (pp. 339-356). Springer Netherlands.

Sibilio, M. (2014). La didattica semplessa, Liguori.

Stella, G. (2003). La valutazione della dislessia: un approccio neuropsicologico [Evaluating dyslexia: a neuropsychological approach]. Città aperta.

Stella, G. (2004). La dislessia, II mulino.

Tressoldi, P. E., Stella, G., \& Faggella, M. (2001). The development of reading speed in Italians with dyslexia a longitudinal study. Journal of learning disabilities, 34(5), 414-417.

Vio, C.(2013). Dislessia e altri DSA a scuola. Strategie efficaci per gli insegnanti [Dyslexia and other SLDs at school. Efficient strategies for teachers], Centro Studi Erickson. 